\title{
Fluctuation conductivity in superconductors in strong electric fields
}

\author{
Todor Mishonov, ${ }^{a, b *}$ Anna Posazhennikova ${ }^{a}$ and Joseph Indekeu ${ }^{a}$ \\ ${ }^{a}$ Laboratorium voor Vaste-Stoffysica en Magnetisme, Katholieke Universiteit Leuven, \\ Celestijnenlaan 200 D, B-3001 Leuven, Belgium \\ ${ }^{b}$ Department of Theoretical Physics, Faculty of Physics, Sofia University St. Kliment Ohridski, \\ 5 J. Bourchier Blvd., 1164 Sofia, Bulgaria
}

\begin{abstract}
We study the effect of a strong electric field on the fluctuation conductivity within the timedependent Ginzburg-Landau theory for the case of arbitrary dimension. Our results are based on the analytical derivation of the velocity distribution law for the fluctuation Cooper pairs, from the Boltzmann equation. Special attention is drawn to the case of small nonlinearity of conductivity, which can be investigated experimentally. We obtain a general relation between the nonlinear conductivity and the temperature derivative of the linear Aslamazov-Larkin conductivity, applicable to any superconductor. For the important case of layered superconductors we derive an analogous relation between the small nonlinear correction for the conductivity and the fluctuational magnetoconductivity. On the basis of these relations we provide new experimental methods for determining both the lifetime constant of metastable Cooper pairs above $T_{c}$ and the coherence length. A systematic investigation of the 3rd harmonic of the electric field generated by a harmonic current can serve as an alternative method for the examination of the metastable Cooper-pair relaxation time.
\end{abstract}

\section{INTRODUCTION}

Fluctuation phenomena in superconductors have been intensively studied for more than 3 decades since the discovery of the fluctuation smearing of the superconducting transition. The interest in fluctuations was resurrected when the high-temperature superconductors (HTSC) were found. In conventional BCS-like superconductors the transition temperature $T_{c}$ marks a sharp dividing point between distinct regions of 'superconducting' and 'normal' behavior, and critical fluctuations are almost unobservable. Largely due to their high temperature, small coherence length and quasitwo-dimensional nature, HTSC show a significant smearing of the critical transition and thus the effect of fluctuations in the critical region of copper-oxides is much more pronounced and experimentally accessible. The conductivity, the specific heat, the diamagnetic susceptibility, the thermopower etc. have been observed to increase considerably in the normal state in the vicinity of the transition temperature in cuprate compounds. Importantly, for high-quality HTSC samples the interactions between fluctuations are negligibly small, fluctuations can be described as Gaussian ones and their theory is rather simple. As a result nowadays the study of fluctuation phenomena in superconductors occupies an essential and significant part of the whole physics of superconductivity. For a contemporary review we refer to the work of Larkin and Varlamovi; cf. also the classical review by Skocpol and Tinkhame and the recent review by Mishonov and Peneve especially devoted to Gaussian fluctuations in layered superconductors.

In this paper we are concerned with the effect of fluctuations of the superconducting order parameter on both the linear and the nonlinear conductivity near and above the bulk superconducting critical temperature. In order to account for the fluctuation conductivity (or paraconductivity) one needs to apply the time-dependent generalization of the Ginzburg-Landau theory (TDGL). The theoretical study of fluctuation conductivity dates from the paper of Aslamazov and Larkin in 1968 based on the microscopic approach. Although a long time has elapsed, nonlinear effects have not been sufficiently investigated and the theoretical results obtained so far require specification.

The aim of the present paper is to study the kinetics of the superconducting order parameter in a strong electric field within a simple phenomenological approach and to provide new experimental methods for determining such important material constants of superconductors as the lifetime of fluctuation Cooper pairs $\tau_{0}$ and the coherence length $\xi(0)$.

We adopt the ideology of TDGL theory and show that the velocity distribution law of fluctuation Cooper pairs can be derived from a Boltzmann equation. The cases of superconducting wires, thin films, bulk superconductors and striped and layered superconducting systems are analyzed. We are particularly interested in the effects of nonlinearity

*Corresponding author: phone: (+32 16) 327 183, fax.: (+32 16) 327 983, e-mail: todor.mishonov@fys.kuleuven.ac.be 
of conductivity, which can be observed experimentally. We show that the strong critical behavior in the vicinity of $T_{c}$ of the third harmonic of the electric field generated by a harmonic current, can be used as an experimental technique for the determination of the metastable Cooper pair lifetime constant.

This paper is organized as follows. In Section II we discuss the kinetic Boltzmann equation for fluctuation Cooper pairs in strong electric fields and present the momentum distribution law for Cooper pairs in the normal phase and the expression for the fluctuation current. Section III is mainly technical and devoted to dimensionless notations we introduce to make our calculation less complicated. The derivation of the time evolution of the distribution function using canonical variables is given in the Appendix. Section IV explains the paraconductivity in layered materials, such as for example HTSC. We provide the analytical derivation of the conductivity correction in the normal phase in a Lawrence-Doniach superconductor. In Section $\mathrm{V}$ the Aslamazov-Larkin conductivity dependent on the electric field is described in detail for one-, two- and three-dimensional superconductors. We study strong electric field effects on conductivity as well as weak electric field influences below the critical temperature. The interesting case of a striped superconductor, which is becoming very popular nowadays, and that of a thick film can be found in Section VI. The conductivity of such a superconductor depending on the thickness of the film, is obtained. The expressions for the nonlinear conductivity correction can be used for experimental data processing for extracting the lifetime constant of Cooper pairs, as is shown in Section VII. A new experimental technique for probing $\tau_{0}$, based on suggested measurements of the third harmonic of the electric field, can be found in Section VIII. Finally, a discussion and conclusions are given in the last section.

\section{BOLTZMANN EQUATION AND FORMULA FOR THE CURRENT}

As was already mentioned in Section I, the Ginzburg-Landau theory serves as an adequate tool for describing the fluctuation conductivity phenomena in superconductors. The important fundamental constant of the TDGL theory is $\tau_{0}$, which is proportional to the lifetime of metastable Cooper pairs in the normal state:

$$
\tau(\epsilon)=\frac{\tau_{0}}{\epsilon},
$$

where $\epsilon \equiv\left(T-T_{c}\right) / T_{c}$.

Within the weak-coupling BSC theory in the case of negligible depairing mechanisms the temperature-independent constant $\tau_{0}$ satisfies the relation:

$$
\tau_{0}^{(\mathrm{BCS})}=\frac{\pi}{16} \frac{\hbar}{k_{B} T_{c}} .
$$

At present the experimental value for $\tau_{0}$, obtained for the layered cuprate superconductors, is in good agreement with the BCS theory3. From an experimental point of view it is convenient to introduce the dimensionless ratio:

$$
\tau_{\text {rel }}=\frac{\tau_{0}}{\tau_{0}^{(\mathrm{BCS})}},
$$

which just characterizes the deviations of the experimental value from that which is theoretically derived from the microscopic theory for the case of small coupling and negligible depairing. Before establishing the relation between experimental results on fluctuation conductivity and the lifetime constant $\tau_{0}$ the important question to be clarified is what the momentum or velocity distribution of the metastable Cooper pair actually is.

The charge of the Cooper pair is $\left|e^{*}\right|=2|e|$ and its effective mass $m^{*}$ is unambiguously experimentally accessible through many principally different methods: electrostafic charge modulation of the kinetic induftance 6 , surface Hall effect reliable, determination of the thermodynamic equilibrium electric potential related to the Bernoulli potential1 10 . The velocity of a pair along the $x$-direction reads

$$
v_{x}=\frac{p_{x}}{m_{a}^{*}}
$$

and along the $y$ and $z$ directions the velocities are correspondingly $v_{y}=p_{y} / m_{b}^{*}$, and $v_{z}=p_{z} / m_{c}^{*}$. Note that we allow for the presence 0 mass anisotropy.

It was shown 112 within the general TDGL theory that, surprisingly, the momentum distribution law for the metastable Cooper pair is described by the classical Boltzmann equation introduced in physics in 1876 long before the electron was discovered. Nowadays this equation is discussed in many well-known textbooks on kinetics and solid 
state physics 1 . For a detailed description of the relaxation time approximation to the Boltzmann equation see also a well-known textbook 3 . The Boltzmann equation in our context is given by

$$
\left(\frac{\partial}{\partial t}+e^{*} \mathbf{E} \cdot \frac{\partial}{\partial \mathbf{p}}\right) n(\mathbf{p}, t)=-\frac{n(\mathbf{p}, t)-\bar{n}_{p}}{\tau_{p}}=-\frac{n_{p}(t)}{\tau_{p}}+\frac{n_{T}}{\tau_{0}} .
$$

This kinetic equation for fluctuation Cooper pairs has been applied to the fluctuation Hall effect in thin superconducting films 11 and to the fluctuation paraconductivity within the framework of time-dependent Ginzburg-Landau theory 12 , cf. also Ref.1. In Eq.(2.5) $n_{p}(t)=n(\mathbf{p}, t)$, and we introduce the dimensionless number $n_{T}=k_{B} T / a_{0}$, where

$$
a_{0}=\frac{\hbar^{2}}{2 m_{a}^{*} \xi_{a}^{2}(0)}=\frac{\hbar^{2}}{2 m_{b}^{*} \xi_{b}^{2}(0)}=\frac{\hbar^{2}}{2 m_{c}^{*} \xi_{c}^{2}(0)}
$$

is in fact proportional to the first coefficient in the Ginzburg-Landau expansion

$$
a(\epsilon)=a_{0} \epsilon
$$

and

$$
\bar{n}_{p}=\frac{k_{B} T}{\varepsilon_{p}-\mu}
$$

is a standard equilibrium distribution, where the energy spectrum of the layered superconductor with layer spacing $s$ is given by

$$
\varepsilon_{p}=\frac{p_{x}^{2}}{2 m_{a}^{*}}+\frac{p_{y}^{2}}{2 m_{b}^{*}}+\frac{\hbar^{2}}{m_{c}^{*} s^{2}}\left[1-\cos \left(\frac{s p_{z}}{\hbar}\right)\right],
$$

and the chemical potential is defined through $-\mu(T)=a(\epsilon)$. The momentum-dependent relaxation time $\tau_{p}$ obtained 112 by TDGL theory reads as

$$
\tau_{p}=\frac{\tau_{0} a_{0}}{\varepsilon_{p}+a(\epsilon)} .
$$

Let us assume that the electric field is constant and applied along the $x$-direction in the $a b$-plane: $\mathbf{E}=\left(E_{x}, 0,0\right)=$ const. In this case from the Boltzmann equation (2.5) follows the stationary momentum distribution law

$$
\begin{aligned}
n\left(p_{x} ; \varepsilon_{\perp}, E_{x}, T\right)= & \frac{k_{B} T}{\hbar^{2} / 2 m_{a}^{*} \xi_{a}^{2}(0)} \int_{0}^{\infty} \exp \left\{-\left[\left(\frac{\xi_{a}(0) p_{x}}{\hbar}\right)^{2}+\frac{\varepsilon_{\perp}}{\hbar^{2} / 2 m_{a}^{*} \xi_{a}^{2}(0)}+\epsilon\right] \frac{t}{\tau_{0}}\right\} \\
& \times \exp \left\{+\frac{e^{*} E_{x} \xi_{a}(0)}{\hbar / \tau_{0}} \frac{\xi_{a}(0) p_{x}}{\hbar}\left(\frac{t}{\tau_{0}}\right)^{2}-\frac{1}{3}\left(\frac{e^{*} E_{x} \tau_{0}}{\hbar / \xi_{a}(0)}\right)^{2}\left(\frac{t}{\tau_{0}}\right)^{3}\right\} \frac{d t}{\tau_{0}},
\end{aligned}
$$

where

$$
\varepsilon_{\perp}\left(p_{y}, p_{z}\right)=\frac{p_{y}^{2}}{2 m_{b}^{*}}+\frac{\hbar^{2}}{m_{c}^{*} s^{2}}\left[1-\cos \left(\frac{s p_{z}}{\hbar}\right)\right]
$$

and the physical meaning of the dummy parameter $t$ as time is revealed in the Appendix. For zero field $E_{x}=0$ from (2.11) we naturally obtain

$$
\bar{n}\left(p_{x}, \varepsilon_{\perp}\right)=\frac{k_{B} T / a_{0}}{\left(\xi_{a}(0) p_{x} / \hbar\right)^{2}+\varepsilon_{\perp} / a_{0}+\epsilon} .
$$

Once we have the momentum distribution law for the fluctuation Cooper pair, we can immediately proceed to the expression for the stationary fluctuation current for arbitrary dimension $D$ :

$$
\mathbf{j}=\sum_{p} e^{*} \mathbf{v}_{p} \frac{n_{p}}{\mathcal{V}}=\int e^{*} \mathbf{v}_{p} n(\mathbf{p}) \frac{d^{D} p}{(2 \pi \hbar)^{D}}, \quad \mathbf{v}_{p}=\frac{\partial \varepsilon_{p}}{\partial \mathbf{p}}
$$


where $\mathcal{V}=L_{x} L_{y} L_{z}$ is the volume of a system. For the current along the $x$-direction in a layered superconductor we have

$$
\begin{aligned}
j_{x}= & e^{*} \frac{k_{B} T}{\hbar^{2} / 2 m_{a}^{*} \xi_{a}^{2}(0)} \int_{0}^{\infty} \frac{d t}{\tau_{0}} \exp \left\{-\frac{1}{3}\left(\frac{e^{*} E_{x} \tau_{0}}{\hbar / \xi_{a}(0)}\right)^{2}\left(\frac{t}{\tau_{0}}\right)^{3}\right\} \int_{-\pi \hbar / s}^{\pi \hbar / s} \frac{d p_{z}}{2 \pi \hbar} \int_{-\infty}^{\infty} \frac{d p_{y}}{2 \pi \hbar} \\
& \times \exp \left\{-\left[\epsilon+\left(\frac{p_{y} \xi_{b}(0)}{\hbar}\right)^{2}+\left(\frac{2 \xi_{c}(0)}{s} \sin \frac{s p_{z}}{2 \hbar}\right)^{2}\right] \frac{t}{\tau_{0}}\right\} \\
& \times \int_{-\infty}^{\infty} \frac{p_{x}}{m_{a}^{*}}\left\{-\frac{t}{\tau_{0}}\left(\frac{\xi_{a}(0) p_{x}}{\hbar}\right)^{2}+\frac{e^{*} E_{x} \xi_{a}(0)}{\hbar / \tau_{0}}\left(\frac{t}{\tau_{0}}\right)^{2} \frac{\xi_{a}(0) p_{x}}{\hbar}\right\} \frac{d p_{x}}{2 \pi \hbar}=\sigma_{x x} E_{x} .
\end{aligned}
$$

A similar formpla for the current was presented by Gor'kov 13 and modified for layered superconductors by Varlamov and Reggiani14.

\section{DIMENSIONLESS VARIABLES}

To make our further calculations a bit less cumbersome we introduce a number of dimensionless variables: $k_{x}=$ $\xi_{a}(0) p_{x} / \hbar$ and $k_{y}=\xi_{b}(0) p_{y} / \hbar \in(-\infty, \infty)$ are dimensionless momenta, $\theta=s p_{z} / \hbar \in(-\pi, \pi)$ is the Josephson phase and $u=t / \tau_{0} \in(0, \infty)$ is in fact the renormalized time. We also introduce

$$
w \equiv \frac{\varepsilon_{\perp}}{a_{0}}=\left(\frac{p_{y} \xi_{b}(0)}{\hbar}\right)^{2}+\left(\frac{2 \xi_{c}(0)}{s} \sin \frac{s p_{z}}{2 \hbar}\right)^{2}=k_{y}^{2}+\omega(\theta),
$$

where for the well-known Lawrence Doniach model $\omega(\theta)=\varepsilon_{z}\left(p_{z}\right) / a_{0}=\frac{1}{2} r(1-\cos \theta)=r \sin ^{2} \frac{\theta}{2}$. The factor $r=$ $\left(2 \xi_{c}(0) / s\right)^{2}$ originates from the parametrization of the effective mass in $c$-direction, $m_{c}^{*}$, for an anisotropic GL model.

It is convenient to deal with some dimensionless variable $f$, proportional to the electric field

$$
f=\frac{e^{*} E_{x} \tau_{0}}{\hbar / \xi_{a}(0)}
$$

and with the parameter $g$, proportional to the nonlinear electric field correction

$$
g=\frac{f^{2}}{12}=\frac{1}{12}\left(\frac{e^{*} E_{x} \xi_{a}(0)}{\hbar / \tau_{0}}\right)^{2} .
$$

With the above notations we can now rewrite our expression (2.11) for the momentum distribution of the metastable Cooper pair in the layered superconductor:

$$
n\left(k_{x} ; w, f, \epsilon\right)=n_{T} \int_{0}^{\infty} \exp \left[-\left(k_{x}^{2}+\epsilon+w\right) u+f k_{x} u^{2}-\frac{1}{3} f^{2} u^{3}\right] d u
$$

This function is a solution of the static Boltzmann equation which can be written in the form $\left(k=k_{x}\right)$

$$
f \frac{d n(k)}{d k}=-\left(k^{2}+\epsilon+w\right) n(k)+n_{T}
$$

Let us mention some details concerning the derivation of expression (3.4). Direct solution of the Boltzmann equation gives

$$
\begin{array}{r}
n(k)=\exp \left(-\frac{k^{3} / 3+(\epsilon+w) k}{f}\right)\left[n_{T} \int_{k_{0}}^{k} \exp \left(\frac{\tilde{k}^{3} / 3+(\epsilon+w) \tilde{k}}{f}\right) \frac{d \tilde{k}}{f}\right. \\
\left.+n_{0} \exp \left(\frac{k_{0}^{3} / 3+(\epsilon+w) k_{0}}{f}\right)\right] .
\end{array}
$$

¿From here it is easy to see that the boundary condition is $n\left(k_{0}\right)=n_{0}$. Then writing $\tilde{k}=k-u f$, introducing $u=(k-\tilde{k}) / f$ and using 


$$
\frac{\tilde{k}^{3}-k^{3}}{3 f}=-k^{2} u+f k u^{2}-\frac{1}{3} f^{2} u^{3}
$$

we obtain from Eq.(3.6)

$$
\begin{aligned}
n(k)= & n_{T} \int_{0}^{\left(k-k_{0}\right) / f} \exp \left[-\left(k^{2}+\epsilon+w\right) u+f k u^{2}-\frac{1}{3} f^{2} u^{3}\right] d u \\
& +n_{0} \exp \left(-\frac{k^{3} / 3+(\epsilon+w) k}{f}\right) \exp \left(\frac{k_{0}^{3} / 3+(\epsilon+w) k_{0}}{f}\right) .
\end{aligned}
$$

In the limit $k_{0} \rightarrow-\infty$ for $f>0$ or $k_{0} \rightarrow \infty$ for $f<0$ we have $n(-\infty)=n(\infty)=0=n_{0}$ and we arrive at Eq.(3.4). Finally the expression for the current Eq. (2.15) in our notations for the layered superconductor takes the form

$$
\begin{aligned}
j_{x}= & e^{*} \frac{2 k_{B} T}{\hbar} \xi_{a}(0) \int_{0}^{\infty} d u \exp \left\{-\frac{1}{3} f^{2} u^{3}\right\} \int_{-\pi}^{\pi} \frac{d \theta}{2 \pi s} \int_{-\infty}^{\infty} \frac{d k_{y}}{2 \pi \xi_{b}(0)} \\
& \times \exp \left\{-\left[\epsilon+k_{y}^{2}+\frac{r}{2}(1-\cos \theta)\right] u\right\} \\
& \times \int_{-\infty}^{\infty} k_{x} \exp \left\{-u k_{x}^{2}+f u^{2} k_{x}\right\} \frac{d k_{x}}{2 \pi \xi_{a}(0)}=\sigma_{x x} E_{x} .
\end{aligned}
$$

It is informative to compare this result with previously proposed expressions 1314 . Our further analysis of the fluctuation conductivity is based on this general formula.

\section{PARACONDUCTIVITY IN A LAYERED METAL}

As we already pointed out in Section If the fluctuations should be more important in high-temperature materials due in part to their high transition temperatures and extremely short coherence lengths (on the order of $10 \AA$ ). Thus the study of Gaussian fluctuation effects on the conductivity in layered compounds is indispensable and of primary importance. In this section we examine in detail the electric field influence on paraconductivity in layered superconductors and derive analytically the correction to the conductivity in the normal phase.

The integration over $k_{x}$ in the current expression (3.9) can be easily performed:

$$
\int_{-\infty}^{\infty} k_{x} \exp \left\{-u k_{x}^{2}+f u^{2} k_{x}\right\} d k_{x}=\frac{1}{2} \sqrt{\pi u} f \exp \left(\frac{1}{4} f^{2} u^{3}\right) .
$$

Hence we have for the current in the layered superconductor the expression

$$
\begin{aligned}
j_{x}= & \frac{e^{*} k_{B} T}{2 \pi^{1 / 2} \hbar} f \int_{-\pi}^{\pi} \frac{d \theta}{2 \pi s} \int_{-\infty}^{\infty} \frac{d k_{y}}{2 \pi \xi_{b}(0)} \int_{0}^{\infty} d u \sqrt{u} \\
& \times \exp \left\{-\left[\epsilon+k_{y}^{2}+\frac{r}{2}(1-\cos \theta)\right] u-\frac{1}{12} f^{2} u^{3}\right\}=\sigma_{x x} E_{x}
\end{aligned}
$$

and, consequently, the fluctuation conductivity reads

$$
\begin{aligned}
\sigma_{x x}= & \frac{2}{\pi^{1 / 2}} \frac{e^{2} k_{B} T \tau_{0} \xi_{a}(0)}{\hbar^{2}} \int_{-\pi}^{\pi} \frac{d \theta}{2 \pi s} \int_{-\infty}^{\infty} \frac{d k_{y}}{2 \pi \xi_{b}(0)} \int_{0}^{\infty} d u \sqrt{u} \\
& \times \exp \left\{-\left[\epsilon+k_{y}^{2}+\omega(\theta)\right] u-g u^{3}\right\}=j_{x} / E_{x} .
\end{aligned}
$$

After integrating over $k_{y}$ we obtain from (4.3):

$$
\sigma_{x x}=\frac{e^{2} k_{B} T \tau_{0} \xi_{a}(0)}{\pi \hbar^{2} s \xi_{b}(0)} \int_{-\pi}^{\pi} \frac{d \theta}{2 \pi} \int_{0}^{\infty} d u \exp \left\{-[\epsilon+\omega(\theta)] u-g u^{3}\right\}=j_{x} / E_{x} .
$$

Thus the conductivity in the Lawrence-Doniach (LD) model can be expressed in the way

$$
\sigma_{\mathrm{LD}}=\frac{e^{2} k_{B} T \tau_{0} \xi_{a}(0)}{\pi \hbar^{2} s \xi_{b}(0)} \int_{0}^{\infty} d u \exp \left\{-\left(\epsilon+\frac{r}{2}\right) u-g u^{3}\right\} \int_{-\pi}^{\pi} \exp \left\{\frac{r}{2} \cos (\theta) u\right\} \frac{d \theta}{2 \pi}
$$


or, more conveniently,

$$
\sigma_{\mathrm{LD}}(\epsilon, g ; r)=\frac{e^{2} k_{B} T \tau_{0} \xi_{a}(0)}{\pi \hbar^{2} s \xi_{b}(0)} \int_{0}^{\infty} d u \exp \left\{-(\epsilon+r / 2) u-g u^{3}\right\} I_{0}(r u / 2),
$$

where

$$
I_{0}(x)=\frac{1}{\pi} \int_{0}^{\pi} \mathrm{e}^{x \cos \theta} d \theta=\sum_{n=0}^{\infty}\left[\frac{(x / 2)^{n}}{n !}\right]^{2}=J_{0}(i x)
$$

is the Bessel function for imaginary argument.

In the case of zero electric field $E_{x}=0$ the integral over $u$ in Eq.(4.6) is easy to compute since

$$
\int_{-\pi}^{\pi} \frac{d \theta}{2 \pi} \int_{0}^{\infty} d u \exp \left\{-\left[\epsilon+\frac{r}{2}(1-\cos \theta)\right] u\right\}=\int_{-\pi}^{\pi} \frac{d \theta}{2 \pi} \frac{1}{\left(\epsilon+\frac{r}{2}\right)-\frac{r}{2} \cos \theta}=\frac{1}{\sqrt{\epsilon(\epsilon+r)}}
$$

and we obtain the Lawrence-Doniach formula for the fluctuation conductivity of a layered superconductor

$$
\sigma_{\mathrm{LD}}(\epsilon ; r)=\frac{e^{2} k_{B} T \tau_{0} \xi_{a}(0)}{\pi \hbar^{2} s \xi_{b}(0)} \frac{1}{\sqrt{\epsilon(\epsilon+r)}} .
$$

Hereafter we define, for simplicity, $\sigma(\epsilon ; r) \equiv \sigma(\epsilon, 0 ; r)$.

It is simpler to deal with the dimensionless function (cf. Varlamov and Reggiani14 Eq. (7))

$$
\varsigma_{\mathrm{LD}}(\epsilon, g ; r)=\frac{\sigma_{\mathrm{LD}}(\epsilon, g ; r)}{\sigma_{\mathrm{LD}}(\epsilon ; r)}=\sqrt{\epsilon(\epsilon+r)} \int_{0}^{\infty} \exp \left\{-(\epsilon+r / 2) u-g u^{3}\right\} I_{0}(r u / 2) d u .
$$

Then Eq. (4.6) reads

$$
\sigma_{\mathrm{LD}}(\epsilon, g ; r)=\sigma_{\mathrm{LD}}(\epsilon ; r) \varsigma_{\mathrm{LD}}(\epsilon, g ; r)
$$

It is important to note that the product of two exponents in 4.10 can be expressed in terms of the third-derivative operator acting on the function of $\epsilon$

$$
\exp \left(-g u^{3}\right) \exp (-\epsilon u)=\exp \left(g \frac{\partial^{3}}{\partial \epsilon^{3}}\right) \exp (-\epsilon u)
$$

This key observation immediately leads us to the simple general relation between the nonlinear fluctuation conductivity and the linear one in evanescent field, $\sigma(\epsilon)$,

$$
\sigma(\epsilon>0, g)=\exp \left(g \frac{\partial^{3}}{\partial \epsilon^{3}}\right) \sigma(\epsilon)
$$

which is similar to the relation for the magnetoconductivity, derived in Ref.3. For our dimensionless function (4.10) we get

$$
\varsigma(\epsilon, g)=\frac{1}{\sigma(\epsilon)} \exp \left(g \frac{\partial^{3}}{\partial \epsilon^{3}}\right) \sigma(\epsilon) .
$$

In the limit $g \rightarrow 0$ the electric-field-dependent conductivity can be written in a simple form

$$
\sigma(\epsilon, f)=\sigma(\epsilon)+\Delta \sigma_{f}(\epsilon, f) \approx\left(1+g \frac{\partial^{3}}{\partial \epsilon^{3}}\right) \sigma(\epsilon)
$$

and thus the nonlinear electric-field correction to the conductivity reads

$$
\Delta \sigma_{f}(\epsilon, f) \approx \frac{f^{2}}{12} \frac{\partial^{3}}{\partial \epsilon^{3}} \sigma(\epsilon) .
$$

Now we can derive the conductivity, dependent on the electric field in the Lawrence-Doniach model of a layered superconductor. In principle we may start either from the right-hand-side of Eq.(4.8) (i.e., we can take the third 
derivative after averaging over the Josephson phase), or we can perform the differentiation before integrating over $\theta$. The latter is a useful method for the evaluation of complicated integrals necessary for experimental data processing and leads to

$$
\oint \frac{d \theta}{2 \pi} \frac{1}{\left[\epsilon+\frac{r}{2}(1-\cos \theta)\right]^{4}}=-\frac{1}{6} \frac{\partial^{3}}{\partial \epsilon^{3}} \frac{1}{\sqrt{\epsilon(\epsilon+r)}}=\frac{\epsilon^{3}+\frac{3}{2} r \epsilon^{2}+\frac{9}{8} r^{2} \epsilon+\frac{5}{16} r^{3}}{[\epsilon(\epsilon+r)]^{7 / 2}} .
$$

Finally for the Lawrence-Doniach conductivity we have

$$
\sigma_{\mathrm{LD}}(\epsilon, g ; r) \approx\left[1-\frac{1}{2} \frac{\epsilon^{3}+\frac{3}{2} r \epsilon^{2}+\frac{9}{8} r^{2} \epsilon+\frac{5}{16} r^{3}}{(\epsilon+r)^{3}} f_{\epsilon}^{2}\right] \sigma_{\mathrm{LD}}(\epsilon ; r), \quad f_{\epsilon} \ll 1,
$$

where

$$
f_{\epsilon}=\frac{\left|e^{*} E_{x}\right| \xi_{a}(\epsilon) \tau(\epsilon)}{\hbar}=\frac{|f|}{\epsilon^{3 / 2}}=\frac{\left|E_{x}\right|}{E_{c}(\epsilon)}, \quad g_{\epsilon}=\frac{1}{12} f_{\epsilon}^{2}=\frac{g}{\epsilon^{3}}
$$

and other notations are

$$
\begin{gathered}
E_{c}(\epsilon)=\frac{\hbar}{\left|e^{*}\right| \xi(\epsilon) \tau(\epsilon)}=E_{c}(0) \epsilon^{3 / 2}, \quad E_{c}(0)=\frac{\hbar}{\left|e^{*}\right| \xi(0) \tau_{0}}, \\
\xi_{a, b, c}(\epsilon)=\frac{\xi_{a, b, c}(0)}{\epsilon^{1 / 2}}, \quad r_{\epsilon}=\frac{r}{\epsilon}=\left(\frac{2 \xi_{c}(\epsilon)}{s}\right)^{2} .
\end{gathered}
$$

Our dimensionless $\varsigma$ function can now be rewritten in the scaled variables, as can be seen from (4.10),

$$
\varsigma_{\mathrm{LD}}(\epsilon, g ; r)=\varsigma_{\mathrm{LD}}\left(1, g_{\epsilon} ; r_{\epsilon}\right) .
$$

In conclusion of this section we obtain the formula for the electric-field-dependent correction to the conductivity in the Lawrence-Doniach model for a layered superconductor:

$$
\Delta \sigma_{f}\left(\epsilon, E_{x}\right)=-\frac{4 k_{B} T e^{4}\left[\xi_{a}(0) \tau_{0}\right]^{3}}{\pi \hbar^{4} s \xi_{b}(0)} \frac{\left[\epsilon^{3}+\frac{3}{2} r \epsilon^{2}+\frac{9}{8} r^{2} \epsilon+\frac{5}{16} r^{3}\right]}{[\epsilon(\epsilon+r)]^{7 / 2}} E_{x}^{2} \equiv \Delta j_{x} / E_{x} .
$$

\section{ASLAMAZOV-LARKIN CONDUCTIVITY FOR D-DIMENSIONAL SUPERCONDUCTORS}

In this section we are concerned with the derivation of the Aslamazov-Larkin electric-field-dependent conductivity for bulk superconductors $(D=3)$, thin films $(D=2)$ and wires $(\mathrm{D}=1)$. The results can be generalized for the case of arbitrary dimension.

We start with a one-dimensional superconductor. In order to derive the expression for the one-dimensional fluctuation conductivity we should set $p_{y}=p_{z}=0$ in (2.9) and exclude from Eq. (4.3) the integration over the perpendicular component of the momenta

$$
\mathbf{p}_{\perp}=\left(p_{y}, p_{z}\right)=\left(\frac{\hbar k_{y}}{\xi_{b}(0)}, \frac{\hbar \theta}{s}\right)
$$

(the electric field is as usual parallel to the $\mathrm{x}$-direction). We immediately get the final expression

$$
\sigma_{1 \mathrm{D}}=\frac{2}{\pi^{1 / 2}} \frac{e^{2} k_{B} T \tau_{0} \xi_{a}(0)}{\hbar^{2}} \int_{0}^{\infty} \sqrt{u} \exp \left\{-\epsilon u-g u^{3}\right\} d u=\frac{I_{x}}{E_{x}}
$$

For the derivation of a two-dimensional conductivity, induced by the fluctuations, we have to cancel averaging with respect of the Josephson phase $\theta$ in Eq. (4.4) and we find

$$
\sigma_{2 \mathrm{D}}=\frac{e^{2} k_{B} T \tau_{0} \xi_{a}(0)}{\pi \hbar^{2} \xi_{b}(0)} \int_{0}^{\infty} \exp \left\{-\epsilon u-g u^{3}\right\} d u=\frac{j_{x}^{(2 \mathrm{D})}}{E_{x}} .
$$


The case of a layered superconductor turns into the bulk (3D) one in the limit $r \rightarrow \infty$, when the distance between the layers tends to zero. Taking into account the asymptotics for the Bessel function in Eq. (4.6), cf. Ref 14.

$$
I_{0}(x \gg 1) \approx \frac{\mathrm{e}^{x}}{\sqrt{2 \pi x}}, \quad \exp \left(-\frac{r}{2} u\right) I_{0}\left(\frac{r}{2} u\right) \approx \frac{1}{\sqrt{\pi r u}},
$$

we obtain the bulk fluctuation conductivity expression

$$
\sigma_{3 \mathrm{D}}=\frac{e^{2} k_{B} T \tau_{0} \xi_{a}(0)}{2 \pi^{3 / 2} \hbar^{2} \xi_{b}(0) \xi_{c}(0)} \int_{0}^{\infty} \exp \left\{-\epsilon u-g u^{3}\right\} \frac{d u}{\sqrt{u}}=\frac{j_{x}^{(3 \mathrm{D})}}{E_{x}}
$$

These results bring us naturally to the general expression for the fluctuation conductivity for arbitrary dimension $D$ and $T$ above $T_{c}$ :

$$
\sigma_{\mathrm{D}}(\epsilon, g)=\frac{e^{2} k_{B} T \tau_{0} \xi_{a}^{2}(0)}{2^{D-2} \pi^{D / 2} \hbar^{2} \xi^{D}(0)} \int_{0}^{\infty} \exp \left\{-\epsilon u-g u^{3}\right\} \frac{d u}{u^{(D-2) / 2}},
$$

(cf. Dorsey 16 (1991), Eq. (47), for $\epsilon>0$ ).

One can express Eq.(5.6) in a form similar to Eq. (4.11):

$$
\sigma_{\mathrm{D}}(\epsilon, f)=\sigma_{\mathrm{D}}(\epsilon) \varsigma_{\mathrm{D}}\left(g_{\epsilon}\right)
$$

where

$$
\sigma_{\mathrm{D}}(\epsilon)=\frac{4 \Gamma\left(\frac{4-D}{2}\right)}{(4 \pi)^{D / 2}} \frac{e^{2}}{\hbar^{2}} k_{B} T \frac{\tau(\epsilon) \xi_{a}^{2}(\epsilon)}{\xi^{D}(\epsilon)}
$$

is the fluctuation conductivity in zero electric field, and

$$
\xi^{D}(\epsilon) \equiv\left\{\begin{array}{l}
\xi_{a}(\epsilon) \text { for } D=1 \\
\xi_{a}(\epsilon) \xi_{b}(\epsilon) \text { for } D=2 \\
\xi_{a}(\epsilon) \xi_{b}(\epsilon) \xi_{c}(\epsilon) \text { for } D=3 \\
\ldots
\end{array}\right.
$$

The function

$$
\varsigma_{\mathrm{D}}\left(g_{\epsilon}\right)=\frac{1}{\Gamma\left(\frac{4-D}{2}\right)} \int_{0}^{\infty} v^{1-D / 2} \mathrm{e}^{-v-g_{\epsilon} v^{3}} d v, \quad v=\epsilon u
$$

is a dimensionless function containing the electric-field dependence of the fluctuation conductivity.

For small electric field $f_{\epsilon} \ll 1$, in accordance with Eq (4.15), we have:

$$
\varsigma_{\mathrm{D}}\left(f_{\epsilon}\right) \approx 1-\left(\frac{4-D}{2}\right)\left(\frac{6-D}{2}\right)\left(\frac{8-D}{2}\right) \frac{f_{\epsilon}^{2}}{12} .
$$

\section{A. Strong electric field expansion}

In this subsection we study the conductivity analytically in the limit of electric fields that are large compared with the reduced temperature distance to the critical point. To this end we introduce the scaled variable $\epsilon_{f}=12^{1 / 3} \epsilon / f^{2 / 3}$ and focus on the regime of small $\epsilon_{f}$. The sign of $\epsilon_{f}$ is arbitrary. Our analysis applies to $T \leq T_{c}$ as well as $T>T_{c}$. Changing the integration variable $u$ in Eq. (5.6) to $f^{2 / 3} u$ allows us to extract the dominant field dependence at the critical point. Subsequently we use the series expansion of $\exp (-\epsilon u)$ and integrate it term by term for obtaining a series in $\epsilon_{f}$. We arrive at the result

$$
J_{\mathrm{D}}(\epsilon, g) \equiv \int_{0}^{\infty} \exp \left\{-\epsilon u-g u^{3}\right\} \frac{d u}{u^{(D-2) / 2}}=4 \frac{f^{-(4-D) / 3}}{12^{(D+2) / 6}} \sum_{n=0}^{\infty} \frac{\left(-\epsilon_{f}\right)^{n}}{n !} \Gamma\left(\frac{2 n+4-D}{6}\right) .
$$


The convergence is very good for $\left|\epsilon_{f}\right| \leq 1$. For instance, for $\epsilon_{f}=0.2$ the errors in zeroth, first, second and third order are, respectively, 12\%, 1\%,0.07\% and $40 \mathrm{ppm}$ (parts per million). This series leads to the following expression for the conductivity

$$
\sigma_{\mathrm{D}}(\epsilon, f)=\sigma_{\mathrm{D}}(f) \Sigma_{\mathrm{D}}\left(\epsilon_{f}\right)
$$

with

$$
\Sigma_{\mathrm{D}}\left(\epsilon_{f}\right) \equiv 1+\sum_{n=1}^{\infty} \frac{\left(-\epsilon_{f}\right)^{n}}{n !} \frac{\Gamma\left(\frac{2 n+4-D}{6}\right)}{\Gamma\left(\frac{4-D}{6}\right)}
$$

and

$$
\begin{aligned}
\sigma_{\mathrm{D}}(f) & =\frac{\Gamma\left(\frac{4-D}{6}\right) e^{2} k_{B} T \tau_{0} \xi_{a}^{2}(0) f^{-(4-D) / 3}}{3^{(D+2) / 6} 2^{(4 D-10) / 3} \pi^{D / 2} \hbar^{2} \xi^{D}(0)} \\
& =\frac{4 \Gamma\left(\frac{4-D}{6}\right) k_{B} T \tau_{0}^{(D-1) / 3}}{[2 \sqrt{\pi} \xi(0)]^{D} E_{x}^{(4-D) / 3}}\left(\frac{e \xi_{a}(0)}{\sqrt{3} \hbar}\right)^{(D+2) / 3}
\end{aligned}
$$

is the fluctuation conductivity at $T_{c}$; cf 14.16 .

\section{B. Weak electric fields below $T_{c}$}

In order to study the fluctuation conductivity for temperatures slightly below $T_{c}$ and in small electric fields we must take into account that the limit of zero field is singular due to the occurrence of bulk superconductivity. The contribution to the conductivity that we calculate in this subsection must be well separated physically from that due to the onset of bulk order. The approximation scheme we develop here is a good one for relatively weak electric fields, which satisfy at $T<T_{c}$ the condition

$$
f_{\epsilon} \equiv \frac{|f|}{|\epsilon|^{3 / 2}}=\frac{2\left|e E_{x}\right| \xi_{a}(0) \tau_{0}}{\hbar|\epsilon|^{3 / 2}}<1 .
$$

Especially below $T_{c}$ we have to take into account that the temperature distance to the critical point $\epsilon$ is renormalized as can be seen in self-consistent mean-field-like approximations which we will briefly consider later. The origin of this effect lies in the non-linear character of the TDGL equation, which cannot be neglected for high densities of fluctuation Cooper pairs. The selfconsistent approximation decouples the non-linearity, resulting in a linear problem with a modified $\epsilon$, to be denoted by $\epsilon_{r}$. To alleviate the notation we will postpone this substitution until the end of this subsection.

For $T<T_{c}$ we write $\epsilon=-|\epsilon|$ and to the integral in Eq. (5.12) we apply the Gaussian saddle point approximation for weak electric fields. This amounts to looking for the maximum of the argument of the exponential function, since the remaining factor is an algebraic function of $u$ and therefore slowly varying. Defining

$$
F(u)=-|\epsilon| u+\frac{f^{2} u^{3}}{12}
$$

the saddle-point approximation can be written as

$$
\mathrm{e}^{-F(u)} \approx \mathrm{e}^{-F\left(u_{0}\right)} \sqrt{\frac{2 \pi}{F^{\prime \prime}\left(u_{0}\right)}} \delta\left(u-u_{0}\right),
$$

where $u_{0}$ is the minimum of $F(u)$, given by

$$
u_{0}=2 \frac{\sqrt{|\epsilon|}}{|f|}
$$

the time interval $t_{0}=\tau_{0} u_{0}$ has a transparent physical meaning, $\left|e^{*} E_{x}\right| t_{0}=2 \hbar / \xi_{a}(\epsilon)$. In order for this approximation to be accurate, the condition 


$$
F^{\prime \prime}\left(u_{0}\right) u_{0}^{2} \gg 1
$$

must be satisfied, which is equivalent to

$$
f_{\epsilon} \ll 1
$$

This condition can be seen to arise from two requirements. Firstly, already in $D=2$, in the absence of the power of $u$ in the integral, the validity of the saddle-point approximation requires

$$
\exp \left(-\frac{1}{2} F^{\prime \prime}\left(u_{0}\right) u_{0}^{2}\right) \ll 1
$$

in order for the integration interval to be extendable to $(-\infty, \infty)$. Secondly, in the presence of slowly varying additional factors in the integrand, we must check the consistency of the approximation by performing a Taylor expansion of the algebraic function of $u$ about $u_{0}$. If we denote this function by $G(u)$, we may approximate this by the constant $G\left(u_{0}\right)$ in the integral, provided $G(u)$ deviates only weakly from linearity in a neighbourhood of width $w_{F}$ around $u_{0}$, where $w_{F}$ is the standard deviation of the Gaussian function. This is fulfilled when

$$
G^{\prime \prime}\left(u_{0}\right) w_{F}^{2} / G\left(u_{0}\right) \ll 1 .
$$

Since $w_{F}^{2}=1 / F^{\prime \prime}\left(u_{0}\right)$ and $G(u)$ is simply a power of $u$, this condition coincides with Eq. (5.20).

Within the range of validity of the saddle-point approximation we thus arrive at the following result for the integral

$$
J_{\mathrm{D}}(-|\epsilon|, f) \approx \sqrt{\pi}|\epsilon|^{-(4-D) / 2}\left(\frac{2}{f_{\epsilon}}\right)^{(3-D) / 2} \exp \left(\frac{4}{3 f_{\epsilon}}\right), \quad \text { for } \quad \mathrm{e}^{2 / f_{\epsilon}} \gg 1 .
$$

According to Eq. (5.6) and taking into account the renormalization of $\epsilon$ to $\epsilon_{r}$ we obtain

$$
\sigma_{\mathrm{D}}(-|\epsilon|, f) \approx \frac{e^{2} k_{B} T}{2^{(3 D-7) / 2} \pi^{(D-1) / 2} \hbar^{2}} \frac{\tau\left(\epsilon_{r}\right) \xi_{a}^{2}\left(\epsilon_{r}\right)}{\xi^{D}\left(\epsilon_{r}\right)} f_{\epsilon}^{(D-3) / 2} \exp \left(\frac{4}{3 f_{\epsilon}}\right) .
$$

The replacement $\epsilon \rightarrow \epsilon_{r}$ derives from the use of a Maxwell-type selfconsistent approach (cf. Ref.B) for solving the Boltzmann equation. The implicit equation relating the renormalized and bare parameters is given by

$$
\epsilon_{r}-\epsilon=\frac{\mu_{0}}{m_{a b}^{*}}\left[e^{*} \lambda_{a b}(0)\right]^{2} \int \frac{d^{D} p}{(2 \pi \hbar)^{D}} n\left(\mathbf{p}, \mathbf{E}, \epsilon_{r}\right),
$$

with

$$
\frac{1}{\lambda_{a b}^{2}(0)} \equiv-\left.T_{c} \frac{d}{d T} \frac{1}{\lambda_{a b}^{2}(T)}\right|_{T_{c}^{-}}
$$

where details of the procedure of ultraviolet regularization will be presented elsewhere.

The most significant aspect of our result is the dramatic exponential increase in the fluctuation conductivity for small electric fields. Therefore, for $f_{\epsilon} \ll 1$, the fluctuation part becomes of the same order of magnitude as the normal-state background, $\sigma_{D}(-|\epsilon|, f) \simeq \sigma_{N}$, and the fluctuation conductivity will no longer be just a perturbation but a significant part of the total conductivity

$$
\sigma_{\text {tot }}=\sigma_{D}(\epsilon, f)+\sigma_{N}(T)=j_{\text {tot }} / E_{x}
$$

This extraordinary increase of the conductivity natyrally leads to a minimum in the current as a function of the applied field, in agreement with the Gor'kov analysis 13 that the current-voltage characteristic must have a perfectly noticeable section corresponding to negative differential conductivity. At a suitable potential difference between the ends of the film, the generation of radiation should be observed. Indeed, for every $\epsilon<0$ the generation of radiation will start for electric fields lower than the critical one, $E_{\text {gen }}(\epsilon=-|\epsilon|)$, determined by the criterion

$$
\left.\frac{d j_{\mathrm{tot}}\left(E_{x}\right)}{d E_{x}}\right|_{E_{\mathrm{gen}}}=0
$$

which is equivalent to 


$$
\left.f_{\text {gen }} \frac{d}{d f} \sigma(-|\epsilon|, f)\right|_{f_{\text {gen }}}+\sigma\left(-|\epsilon|, f_{\text {gen }}\right)=-\sigma_{N}(T) .
$$

Within the selfconsistent approximation Eq. (5.27) it is easy to obtain theoretical formulae for the case when the fluctuations are nonlinear

$$
E_{\text {gen }}\left(-\left|\epsilon_{r}\right|\right)=E_{c}(0) f_{\text {gen }}\left(-\left|\epsilon_{r}\right|\right),
$$

where the unit of electric field $E_{c}(0)$ is defined in Eq. (4.20), and this will be a nontrivial test of the validity of the selfconsistent approximation applied to the TDGL equation and, following from it, the Boltzmann equation for fluctuation Cooper pairs. Now we are addressing cases important for the applications, in which the fluctuation superconductivity can be easily investigated.

\section{STRIPED SUPERCONDUCTORS AND THICK FILMS}

The latest achievements in nanotechnology provide us in principle with a tool for a practical realization of so called striped superconductors with controlled parameters. For this it is necessary to cut stripes from a superconducting film using some appropriate lithographic technology. The amazing observation about the striped materials is that they are of "intermediate" dimensionality, i.e., they are neither 1-dimensional systems nor 2-dimensional ones. The closer the striped superconductor is to its critical temperature $T_{c}$, the more "perfectly" two-dimensional material it becomes, because the stripes become increasingly coherent. Analogously to the Lawrence-Doniach model for the layered superconductor we can describe this situation in terms of "dimensional crossover". In the present section we show that the fluctuation conductivity longitudinal to the stripes can be derived following the standard procedure for layered superconductors. We have also to mention that probably some underdoped cuprates are naturally striped and this phenomenon has been at the center of the attention attracted by HTSC during the last few years.

It is obvious from Section V, for example, that the LD conductivity for a layered superconductor can be naturally derived from a two-dimensional AL conductivity just by integrating that over the momentum in the direction perpendicular to the plane

$$
\sigma_{\mathrm{LD}}(\epsilon, f)=\int \frac{d p_{z}}{2 \pi \hbar} \sigma_{2 \mathrm{D}}\left(\epsilon+\frac{\varepsilon_{z}\left(p_{z}\right)}{a_{0}}, f\right)=\int_{-\pi}^{\pi} \sigma_{2 \mathrm{D}}\left(\epsilon+\frac{r}{2}(1-\cos \theta), f\right) \frac{d \theta}{2 \pi s} .
$$

Thus the fluctuation conductivity for a striped superconductor reads

$$
\sigma_{\text {striped }}(\epsilon, f)=\frac{1}{s} \oint \sigma_{1 \mathrm{D}}\left(\epsilon+\frac{r}{2}(1-\cos \theta), f\right) \frac{d \theta}{2 \pi},
$$

where $s$ now stands for the period of the stripes.

For a thick film with thickness $d_{\text {film }}$ we have to sum over the discrete spectrum of the energy associated with the motion in z-direction:

$$
\varepsilon_{z}\left(p_{z}\right)=\frac{p_{z}^{2}}{2 m_{c}^{*}}, \quad \frac{\varepsilon_{z}\left(p_{z}\right)}{a_{0}}=\left(\frac{\pi \xi_{c}(0)}{d_{\text {film }}}\right)^{2} n_{z}^{2}
$$

since

$$
p_{z}=\frac{\pi \hbar}{d_{\text {film }}} n_{z}, \quad n_{z}=0,1,2,3, \ldots
$$

In the expression for the fluctuation conductivity of a thick film we have now summation instead of integration

$$
\sigma_{\mathrm{film}}(\epsilon, f)=\frac{1}{d_{\mathrm{film}}} \sum_{p_{z}} \sigma_{2 \mathrm{D}}\left(\epsilon+\varepsilon_{z}\left(p_{z}\right) / a_{0}, f\right)=\frac{1}{d_{\mathrm{film}}} \sum_{n_{z}}^{\infty} \sigma_{2 \mathrm{D}}\left(\epsilon+\left(\pi \xi_{c}(0) / d_{\mathrm{film}}\right)^{2} n_{z}^{2}, f\right),
$$

which for zero electric field can be readily performed using

$$
\sum_{n=0}^{\infty} \frac{1}{a^{2}+b^{2} n^{2}}=\frac{\pi}{2 a b \tanh \left(\frac{\pi a}{b}\right)}+\frac{1}{2 a^{2}} .
$$


Finally we obtain the expression for the 3D conductivity of the film, which interpolates between 2D and 3D behaviour analogously to the conductivity for a layered superconductor:

$$
\begin{gathered}
\sigma_{\text {film }}(\epsilon)=\sigma_{2 D}(\epsilon)\left\{\frac{1}{2 d_{\text {film }}}+\frac{1}{2 \xi_{c}(\epsilon) \tanh \left(\frac{d_{\text {film }}}{\xi_{c}(\epsilon)}\right)}\right\}=\frac{e^{2} \tau_{r e l}}{16 \hbar \epsilon}\left\{\frac{1}{2 d_{\text {film }}}+\frac{\sqrt{\epsilon} \operatorname{coth}\left(\frac{d_{\text {film }} \sqrt{\epsilon}}{\xi_{c}(0)}\right)}{2 \xi_{c}(0)}\right\}, \\
\sigma_{L D}(\epsilon)=\frac{\sigma_{2 \mathrm{D}}(\epsilon)}{\sqrt{s^{2}+\left(2 \xi_{c}(\epsilon)\right)^{2}}}=\frac{e^{2} \tau_{\text {rel }}}{16 \hbar \sqrt{\epsilon^{2} s^{2}+\epsilon\left(2 \xi_{c}(0)\right)^{2}}} .
\end{gathered}
$$

Note that the thick film becomes "rightly" two-dimensional in the vicinity of $T_{c}$, thus the dimensionality of it decreases, whereas for the layered superconductor the dimensionality goes up from $D=2$ to $D=3$ as we approach the critical temprature. In such a way a thick film of a strongly anisotropic layered superconductor, $\xi_{c}(0) \ll s \ll d_{\text {film }}$, can have two 17 dimensional crossovers.

\section{DETERMINATION OF THE LIFETIME CONSTANT $\tau_{0}$}

In the current section we show that conductivity measurements in a strong electric field can serve as a method for probing fundamental properties of superconductors such as the lifetime constant of metastable Cooper pairs $\tau_{0}$ and the coherence length $\xi(0)$. We demonstrate that our theoretical results can be effectively used for experimental data processing and determination of both $\tau_{0}$ and $\xi(0)$.

As a rule in an experiment the temperature dependence of a resistivity $\rho_{\exp }(T)$ is examined. The experimentally measured conductivity is consequently $\sigma_{\exp }=1 / \rho_{\exp }(T)$. The in-plane current, if we take into account the first nonlinear correction, can be written in the general form

$$
j_{x}=\sigma_{\exp } E_{x}-\mathcal{A}(\epsilon) E_{x}^{3},
$$

since the nonlinear correction to the fluctuation conductivity is $\Delta \sigma_{f}=-\mathcal{A} E_{x}^{2}$.

Of primary interest for us are the superconducting films. Let us consider the two-dimensional BCS-like superconductor. In this case the parameter $r$ that determines the effective dimensionality of the superconductor is zero, and the periodicity of the Lawrence-Doniach model $s \rightarrow d_{\text {film }}$ ( $d_{\text {film }}$ is the thickness of the superconducting film). Thus the two-dimensional current is $j_{x}^{(2 \mathrm{D})}=d_{\mathrm{film}} j_{x}$.

¿From the expression for the LD fluctuation conductivity Eq (4.23) it follows:

$$
j_{x}^{(2 \mathrm{D})}=\left(\sigma_{N}(T)+\frac{e^{2}}{16 \hbar} \frac{\tau_{\mathrm{rel}}}{\epsilon}\right) E_{x}-\frac{4 \pi^{2}}{\hbar}\left[\xi_{a b}(0) / k_{B} T\right]^{2} \frac{e^{4} \tau_{\mathrm{rel}}^{3}}{(8 \epsilon)^{4}} E_{x}^{3} .
$$

In order to study the fluctuation effect on conductivity one should plot first of alt the paraconductivity contribution to the resistance $1 /\left(1 / \rho_{\exp }(T)-1 / \rho_{N}(T)\right)$ as a function of $T$ (see for example 18 , where indium/oxide films were examined). For $\mathrm{InO}_{\mathrm{x}}$ films the value of $\tau_{\mathrm{rel}}=1.16$ can be used as a tool for the determination of the in-plane coherence length $\xi_{a b}(0)$.

In the general case of a layered LD superconductor the coefficient in the nonlinear correction to fluctuation conductivity according to Eq. (4.23) reads

$$
\mathcal{A}(\epsilon)=\frac{4 k_{B} T e^{4}\left[\xi_{a}(0) \tau_{0}\right]^{3}}{\pi \hbar^{4} s \xi_{b}(0)} \frac{\left[\epsilon^{3}+\frac{3}{2} r \epsilon^{2}+\frac{9}{8} r^{2} \epsilon+\frac{5}{16} r^{3}\right]}{[\epsilon(\epsilon+r)]^{7 / 2}} .
$$

In this case we have three coherence lengths $\xi_{a}(0), \xi_{b}(0), \xi_{c}(0)$, and the current anisotropy $J_{\max } / J_{\min }$.

In this paper we develop a model-free method for the determination of the lifetime of Cooper pairs $\tau_{0}$ and the coherence length from the experimental results for the fluctuation conductivity. The final result is derived on the basis of the Eq. (4.15) after three-fold integration over some time interval $\left(\epsilon_{1}, \epsilon_{2}\right)$. We obtain the following expression for the lifetime

$$
\tau_{0}=\sqrt{\frac{3}{2}} \frac{\hbar}{\left|e E_{x}\right| \xi_{a}(0)}\left\{\frac{\int_{\epsilon_{1}}^{\epsilon_{2}}\left(\tilde{\epsilon}-\epsilon_{1}\right)^{2}\left[-\Delta \sigma_{f}(\tilde{\epsilon}, f)\right] d \tilde{\epsilon}}{\sigma\left(\epsilon_{1}\right)-\left[\sigma\left(\epsilon_{2}\right)+\left(\epsilon_{1}-\epsilon_{2}\right) \sigma^{\prime}\left(\epsilon_{2}\right)+\frac{1}{2}\left(\epsilon_{1}-\epsilon_{2}\right)^{2} \sigma^{\prime \prime}\left(\epsilon_{2}\right)\right]}\right\}^{1 / 2} .
$$


In (7.4) we have the notations

$$
\sigma^{\prime}(\epsilon)=\frac{\partial}{\partial \epsilon} \sigma(\epsilon), \quad \sigma^{\prime \prime}(\epsilon)=\frac{\partial^{2}}{\partial \epsilon^{2}} \sigma(\epsilon)
$$

and $\epsilon_{2} \simeq 0.2$ determines the upper bound on the temperature below which the fluctuation conductivity can be reliably measured. In order to simplify the formula (7.4) we can make the approximation $\epsilon_{2} \rightarrow \infty$. Since the fluctuation phenomena are negligible already for $T-T_{c} \approx 0.15 T_{c}$ the following asymptotic conditions can be imposed in the limit $\epsilon_{2} \rightarrow \infty$

$$
\sigma\left(\epsilon_{2}\right) \rightarrow 0, \quad \epsilon_{2} \sigma^{\prime}\left(\epsilon_{2}\right) \rightarrow 0, \quad \epsilon_{2}^{2} \sigma^{\prime \prime}\left(\epsilon_{2}\right) \rightarrow 0 .
$$

Finally we obtain the formulae for the lifetime of metastable Cooper pairs and the in-plane coherence length, which can be applied for experimental data processing:

$$
\begin{aligned}
\tau_{0} & =\sqrt{\frac{3}{2}} \frac{\hbar}{\left|e E_{x}\right| \xi_{a}(0)}\left\{\frac{1}{\sigma(\epsilon)} \int_{\epsilon}^{\infty}(\tilde{\epsilon}-\epsilon)^{2}\left[-\Delta \sigma_{f}(\tilde{\epsilon}, f)\right] d \tilde{\epsilon}\right\}^{1 / 2}, \\
\tau_{\mathrm{rel}} & =\sqrt{\frac{3}{2}} \frac{16 k_{B} T}{\pi\left|e E_{x}\right| \xi_{a}(0)}\left\{\frac{1}{\sigma(\epsilon)} \int_{\epsilon}^{\infty}(\tilde{\epsilon}-\epsilon)^{2}\left[-\Delta \sigma_{f}(\tilde{\epsilon}, f)\right] d \tilde{\epsilon}\right\}^{1 / 2}, \\
\xi_{a b}(0) & =\sqrt{\frac{\Phi_{0}}{\pi\left|B_{z}\right|}}\left\{\frac{1}{\sigma(\epsilon)} \int_{\epsilon}^{\infty}(\tilde{\epsilon}-\epsilon)\left[-\Delta \sigma_{h}(\tilde{\epsilon}, h)\right] d \tilde{\epsilon}\right\}^{1 / 4}
\end{aligned}
$$

cf. Ref. 3 Eqs. (201-203). Here

$$
-\Delta \sigma_{h}(\epsilon, h)=\frac{h^{2}}{4} \frac{\partial^{2}}{\partial \epsilon^{2}} \sigma(\epsilon), \quad h \ll \epsilon
$$

is a nonlinear correction to the magnetoconductivity and the magnetic field

$$
h=\frac{B_{z}}{B_{c 2}(0)}
$$

is oriented perpendicular to the $a b$-plane, where

$$
B_{c 2}(0)=-\left.T_{c} \frac{d B_{c 2}(T)}{d T}\right|_{T_{c}}=\frac{\Phi_{0}}{2 \pi \xi_{a b}^{2}(0)}
$$

is the slope of the upper critical field and

$$
\Phi_{0}=\frac{2 \pi \hbar}{\left|e^{*}\right|}
$$

is the magnetic flux quantum.

\section{CONDUCTIVITY CORRECTION BY DETECTION OF 3RD HARMONICS}

As an alternative method for probing the fundamental constants of the BCS theory we suggest the systematic investigation of the third harmonic of the electric field generated by a harmonic current. Third-harmonic measurements are easier to perform than those of resistivity, and, moreover, the effect arising from fluctuations is exceptionally pronounced.

In general $A C$ current response and investigation of higher harmonics is a standard method for investigation of nonlinear effects on superconductivity. A homogeneous electric field

$$
E_{x}(t)=E_{0} \cos (\omega t),
$$

for example, creates a small nonlinear response for the first harmonic and a cubic field dependence of the 3rd harmonic of the current 


$$
\begin{aligned}
j_{x}(t) & =\sigma E_{x}(t)-\mathcal{A} E_{x}^{3}(t) \\
& =j_{1 f} \cos (\omega t)+j_{3 f} \cos (3 \omega t),
\end{aligned}
$$

where for the amplitudes of the harmonics we have

$$
j_{1 f} / E_{0}=\sigma-\frac{3}{4} \mathcal{A} E_{0}^{2}, \quad j_{3 f}=-\frac{1}{4} \mathcal{A} E_{0}^{3} .
$$

If necessary, a smooth analytical normal part of the nonlinear coefficient $\mathcal{A}$ can be subtracted from the experimental data $\mathcal{A}_{\text {exp }}$

$$
\mathcal{A}_{\text {exp }}=\mathcal{A}_{N}(T)+\mathcal{A}(\epsilon), \quad \mathcal{A}_{N}(T)=A+B T+C T^{2},
$$

in order to extract pure fluctuation behaviour from the nonlinear coefficient of the conductivity correction

$$
\Delta \sigma_{f}=-\mathcal{A}(\epsilon) E_{x}^{2}
$$

If we use the so defined conductivity correction $\Delta \sigma_{f}$ the electric field $E_{x}$ is actually cancelled in Eq. (7.7) and we have to use the coefficient $\mathcal{A}(\epsilon)$ in the expression for $\tau_{0}$, i.e.

$$
\tau_{0}=\sqrt{\frac{3}{2}} \frac{\hbar}{|e| \xi_{a}(0)}\left\{\frac{1}{\sigma(\epsilon)} \int_{\epsilon}^{\infty}(\tilde{\epsilon}-\epsilon)^{2} \mathcal{A}(\tilde{\epsilon}) d \tilde{\epsilon}\right\}^{1 / 2} .
$$

There is no doubt that the electric field is a useful tool for a theoretical analysis but for the experimental realization of the suggested method we have to apply a harmonic current and to measure the harmonics of the voltage

$$
I(t)=I_{0} \cos \omega t, \quad U(t)=U_{1 f} \cos \omega t+U_{3 f} \cos 3 \omega t+U_{5 f} \cos 5 \omega t+\ldots
$$

For small current amplitudes used to avoid heating of the sample the voltage response is in first approximation linear and we have Ohm's law for the resistance of the superconductor strip with length $L$, width $w$ and thickness $d_{\text {film }}$,

$$
U_{1 f}=R(T) I_{0}, \quad R(T)=\rho(T) \frac{L}{w d_{\text {film }}}, \quad \rho(T)=\frac{1}{\sigma_{\exp }(T)}, \quad E_{0}=\frac{U_{1 f}}{L}=\frac{\rho(T) I_{0}}{w d_{\text {film }}} .
$$

Then the absence of the 3rd harmonic of the current, $j_{3 f}=0$, according to Eq. (8.2) with $E_{x}(t)=U(t) / L$ gives

$$
\frac{U_{3 f}}{\rho(T) L} \approx \frac{1}{4} \mathcal{A}(\epsilon)\left(\frac{U_{1 f}}{L}\right)^{3}
$$

to lowest order in $I_{0}$, and finally we obtain

$$
\mathcal{A}(\epsilon) \approx 4 \frac{L^{2}}{\rho(T)} \frac{U_{3 f}}{\left(U_{1 f}\right)^{3}}=4 \frac{L^{3}}{w d_{\text {film }}} \frac{I_{0} U_{3 f}}{\left(U_{1 f}\right)^{4}} .
$$

In this way the nonlinear coefficient necessary for the determination of the lifetime constant in Eq. (8.6) can be expressed through the electronically measured current $I_{0}$, voltage amplitudes $U_{3 f}$ and $U_{1 f}$, and the geometrical parameters of the strip $L, w$ and $d_{\text {film }}$. So the suggested experiment can be performed in every laboratory involved in investigations of superconductivity.

Let us describe qualitatively the temperature dependence of the intensity of the 3rd harmonic when the temperature is increased. In the superconducting state the voltage response is negligible and the $3 \mathrm{f}$ signal will appear abruptly when we reach the critical temperature. After a sharp maximum at $T_{c}$ the $3 \mathrm{f}$ signal will decrease with smaller slope and in the normal region the $3 \mathrm{f}$ signal will be small again and created only by the $2 \mathrm{f}$ oscillations of the temperature and the temperature dependence of the resistivity. In short we predict that $U_{3 f}$ will have a $\lambda$-shaped asymmetric critical singularity. The location of this $\lambda$-point provides a new method for the determination of the critical temperature of superconductors based on the properties of fluctuation phenomena. Our selfconsistent theoretical calculation is applicable above $T_{c}$ where $U_{3 f}$ is much smaller than the value at the $\lambda$-point, but still clearly detectable experimentally. 


\section{DISCUSSION AND CONCLUSIONS}

Let us start analyzing the results derived with the momentum distribution Eqs. (2.11) and (2.13). We have a characteristic velocity related to the equilibrium distribution

$$
v_{c}(\epsilon)=\frac{\hbar}{m_{a}^{*} \xi_{a}(\epsilon)}=v_{c}(0) \sqrt{\epsilon}, \quad m_{a}^{*} v_{c}(0)=\frac{\hbar}{\xi_{a}(0)}
$$

and Eq. (2.13) can now be rewritten as

$$
n\left(v_{x}, \varepsilon_{\perp}\right)=\frac{n_{T}}{\left[v_{x} / v_{c}(\epsilon)\right]^{2}+\epsilon+\varepsilon_{\perp} / a_{0}} .
$$

According to the Drude consideration, a small electric field creates a drift velocity

$$
v_{\mathrm{drift}}(\epsilon)=\frac{e^{*} E_{x} \tau(\epsilon)}{m_{a}^{*}}=\frac{e^{*} E_{x} \tau_{0}}{m_{a}^{*} \epsilon}
$$

and the dimensionless electric field is just the ratio of those two velocities,

$$
f_{\epsilon}=\frac{\left|v_{\mathrm{drift}}(\epsilon)\right|}{v_{c}(\epsilon)}, \quad v_{c}(\epsilon) \simeq \sqrt{\epsilon} v_{\text {Fermi }} \exp \left(-\frac{1}{\rho_{\text {Fermi }} V_{\text {pairing }}}\right) .
$$

¿From a microscopic point of view the characteristic thermal velocity $v_{c}(\epsilon)$ is proportional to the Fermi velocity times the small parameter of the BCS theory, the famous exponent which contains the density of states at the Fermi level and the matrix element of the pairing interaction. This order of magnitude estimation is applicable to anisotropic gaps as well. In addition we have a critical slowing down multiplier $\sqrt{\epsilon}$. Those two factors significantly decrease the characteristic velocity and make possible the experimental observation of the electric-field correction to the fluctuation conductivity proportional to $f_{\epsilon}^{2}$. For normal metals the ratio $v_{\mathrm{drift}}^{2} / v_{\mathrm{Fermi}}^{2} \ll 1$ is extremely small and only AC oscillations of the temperature $T(t)$, mainly 2 , can create harmonics in the voltage response. This effect should also be carefully taken into account for cuprate films for which the thermal resistance between the substrate and the film can be very high; this will be the subject of another work.

Let us also consider in short the $\omega \tau$-quasiparticle criterion to check whether or not Cooper pairs are quasiparticles in the usual sense of condensed matter physics, i.e., $\tau_{p}\left(\epsilon_{p}-\mu\right) / \hbar \gg 1$. For $\mathbf{p}=0$ taking the microscopic value for the lifetime we have

$$
\tau(\epsilon) \frac{a(\epsilon)}{\hbar}=\tau_{0}^{(\mathrm{BCS})} \frac{a_{0}}{\hbar}=\frac{\pi}{16} \frac{a_{0}}{k_{B} T_{c}}=\frac{\pi}{16} \frac{1}{n_{T}} \ll 1 .
$$

This strong inequality means that fluctuation Cooper pairs are not quasiparticles. The notion of Cooper pairs is only a language to describe the properties of slowly decaying diffusion modes of the superconducting order parameter above $T_{c}$. Analogously the "mean free path" $l(\epsilon)=v(\epsilon) \tau(\epsilon)$ is also very short relative to the correlation radius:

$$
\frac{l(\epsilon)}{\xi(\epsilon)}=\frac{v(\epsilon) \tau(\epsilon)}{\xi(\epsilon)}=\frac{\hbar}{m^{*} \xi(\epsilon)} \frac{\tau(\epsilon)}{\xi(\epsilon)}=2 \frac{a_{0} \tau_{0}}{\hbar}=\frac{\pi}{8} \frac{1}{n_{T}} \ll 1
$$

As an illustration let us take a set of parameters corresponding to a high- $T_{c}$ cuprate: $T_{c}=90 \mathrm{~K}, k_{B} T_{c}=7.76 \mathrm{meV}$, $m_{a b}^{*}=11 m_{e}$, where $m_{e}$ is the mass of a free electron, $\xi_{a b}(0)=11 \AA$. Then $a_{0}=\hbar^{2} / 2 m_{a b}^{*} \xi_{a b}^{2}(0)=2.86 \mathrm{meV}$ and $n_{T}=k_{B} T_{c} / a_{0}=2.71>1$. The last inequality ensures that $n(\epsilon)=n_{T} / \epsilon \gg 1$ and this condition of applicability of Rayleigh-Jeans statistics justifies the treatment of the Ginzburg-Landau $\Psi$-function as a classical complex field.

In view of the new effects which can be preficted using the derived velocity distribution we consider the possibility of supercooling of the normal phase, cf. Ref.13, to be very interesting. In this case the fluctuation conductivity could create a negative differential conductivity, which opens perspectives for many technical applications. In order to prevent the nucleation of superconductivity from regions where the current densities and electric fields are very small, depairing impurities should be introduced in the contact area of the microbridge. This could be realized, for example, by evaporation of $\mathrm{Ni}$ on the wide area of the $\mathrm{Al}$ microbridge or by $\mathrm{Mn}$ ions in cuprate films. In both cases the central narrow region of the microbridge should be protected. In a sample prepared under these conditions the criterion of negative differential conductance can be easily satisfied and so the predicted generation of oscillations would probably be the best example of significant fluctuation effects in superconductors. 
Whether the threshold for the generation regime can be described within the nonlinear theory or whether we need to calculate the fluctuation density and the renormalized temperature $T_{r}=T_{c}\left(\epsilon_{r}+1\right)$ in a selfconsistent way, depends on the numerical value of the Ginzburg number. In any case the analyzed solution of the Boltzmann equation suffices to predict a cross-over from positive to negative differential conductivity, as the field decreases. As a precursor of oscillations, when the electric field is decreased, due to strong nonohmic behaviour and low dissipation, the sample will be an excellent frequency mixer. The incipient bulk conductivity should always be taken into account, because the supercooled normal state is metastable and applying a voltage to the superconducting state leads as a rule to a space- and time-inhomogeneous phase.

Some words should be added concerning the history of the kinetic equation introduced in 1876 by Boltzmann. This was the first use of probability concepts in a dynamical theory but the real recognition of the Boltzmann equation was stimulated by the electronic industry in the second half of the 20th century. Indeed, the Boltzmann equation is an essential tool for understanding how electronic devices work.

In sum, in our paper we have used the Boltzmann equation not only as a didactical instrument but also as a means of deriving new results. The Boltzmann equation for fluctuation Cooper pairs is a consequence of the time-dependent Ginzburg-Landau equation. This equation has been derived from microscopic theory and in this sense the Boltzmann equation is a tool for the application of the microscopic theory of superconductivity. That is why the Boltzmann equation can be used to predict the results of new experiments, to help in their interpretation, and even to correct some previously obtained results derived in the frame-work of microscopic theory.

\section{ACKNOWLEDGMENTS}

We highly appreciate the interesting discussion of our paper with A. Varlamov and his stimulating comments. This research has been supported by the Belgian DWTC, IUAP, the Flemish GOA and VIS/97/01 Programmes. A.I.P. is KUL Junior Fellow (F/99/045) and T.M.M. is KUL Senior Fellow (F/00/038).

\section{APPENDIX}

In the Boltzmann equation Eq. (2.5) the decay rate of fluctuation Cooper pairs depends on the kinetic energy $\varepsilon(\mathbf{p})$. As an illustration here we will analyze the isotropic GL model with

$$
\frac{1}{\tau(\mathbf{p})}=\frac{1}{\tau_{0}} \frac{\varepsilon(\mathbf{p})+a_{0} \epsilon}{a_{0}}, \quad \varepsilon(\mathbf{p})=\frac{\mathbf{p}^{2}}{2 m^{*}}, \quad \mathbf{p}=m^{*} \mathbf{v}, \quad \mathbf{v}=\frac{\partial \varepsilon(\mathbf{p})}{\partial \mathbf{p}} .
$$

The Boltzmann equation is a dynamic equation and we consider it natural to trace the time evolution of the velocity distribution starting from some arbitrary initial distribution

$$
n(\mathbf{p}, t=0)=n_{0}(\mathbf{p}) .
$$

As a rule dynamic problems can be more elegantly analyzed using canonical variables. That is why we will analyze the momentum distribution $N(\mathbf{P}, t)$ defined with respect to the canonical momentum

$$
\mathbf{P}=\mathbf{p}+e^{*} \mathbf{A} .
$$

For a constant and space-homogeneous electric field $\mathbf{E}=$ const in the $\varphi=0$ gauge we have

$$
\mathbf{A}(t)=-t \mathbf{E}, \quad \mathbf{A}(t=0)=0, \mathbf{P}(\mathbf{p}, t) \equiv \mathbf{p}-e^{*} \mathbf{E} t,\left(\frac{\partial \mathbf{P}}{\partial \mathbf{p}}\right)_{t}=\mathbb{1}_{D \times D},\left(\frac{\partial \mathbf{P}}{\partial t}\right)_{p}=-e^{*} \mathbf{E} .
$$

Now we will solve the Boltzmann equation using the distribution $N(\mathbf{P}, t)$. The physical quantity is the same - the number of Cooper pairs living at some momentum point - but mathematically the functions are different and their correspondence is given by

$$
N(\mathbf{P}, t) \equiv n(\mathbf{p}, t) .
$$

After this change of the variables the sum of partial derivatives on the left-hand-side of the Boltzmann equation reduces to a usual derivative 


$$
\begin{aligned}
\left(\frac{\partial}{\partial t}+e^{*} \mathbf{E} \cdot \frac{\partial}{\partial \mathbf{p}}\right) N(\mathbf{P}(\mathbf{p}, t), t) & =\frac{\partial N}{\partial t}+\frac{\partial N}{\partial \mathbf{P}} \cdot \frac{\partial \mathbf{P}}{\partial t}+\frac{\partial N}{\partial \mathbf{P}} \cdot \frac{\partial \mathbf{P}}{\partial \mathbf{p}} \cdot e^{*} \mathbf{E} \\
& =\frac{\partial N(\mathbf{P}, t)}{\partial t}=\frac{d N_{P}(t)}{d t} .
\end{aligned}
$$

The physical interpretation is very simple, in an external electromagnetic field the canonical momentum is conserved and reduces simply to a label with which the distribution can be parametrized. In this derivation it is essential to note that when a partial derivative is taken with respect to one argument, the other argument of the function is kept constant. As a consequence, $(\partial n / \partial t)_{p} \neq(\partial N / \partial t)_{P}$. Taking into account that the decay rate and the equilibrium distribution are functions of the kinetic momentum $\mathbf{p}=\mathbf{P}-e^{*} \mathbf{A}$, the Boltzmann equation takes the form

$$
\frac{d N_{P}(t)}{d t}=-\frac{N_{P}(t)-\bar{n}\left(\mathbf{P}-e^{*} \mathbf{A}\right)}{\tau\left(\mathbf{P}-e^{*} \mathbf{A}\right)}=-\left[\frac{\left(\mathbf{P}+e^{*} \mathbf{E} t\right)^{2}}{2 m^{*}}+a_{0} \epsilon\right] \frac{N_{P}(t)}{a_{0} \tau_{0}}+\frac{n_{T}}{\tau_{0}} .
$$

Introducing now dimensionless time and dimensionless canonical momentum

$$
\tilde{u}=\frac{t}{\tau_{0}}, \quad \mathbf{q}=\xi(0) \mathbf{P} / \hbar, \quad q=k-f \tilde{u},
$$

we find that in the $1 \mathrm{D}$ case the Boltzmann equation takes the convenient form

$$
\frac{d N_{P}(\tilde{u})}{d \tilde{u}}=-\left[(q+f \tilde{u})^{2}+\epsilon\right] N_{P}(\tilde{u})+n_{T} .
$$

One can easily check that the function

$$
\begin{aligned}
N_{q}(\tilde{u}) & =n_{T} \exp \left\{-\frac{1}{3 f}(q+f \tilde{u})^{3}-\epsilon \tilde{u}\right\} \int_{0}^{\tilde{u}} \exp \left\{\frac{1}{3 f}\left(q+f u^{\prime}\right)^{3}+\epsilon u^{\prime}\right\} d u^{\prime} \\
& +n_{0}(q) \exp \left\{-\frac{1}{3 f}\left[(q+f \tilde{u})^{3}-q^{3}\right]-\epsilon \tilde{u}\right\}
\end{aligned}
$$

satisfies this ordinary linear differential equation with the initial condition

$$
N_{q}(\tilde{u}=0)=n_{0}(q) .
$$

Now introducing a new dimensionless variable

$$
u \equiv \tilde{u}-u^{\prime}
$$

we find for the distribution $n_{k}(\tilde{u}) \equiv N_{q}(\tilde{u})$ with respect to the kinetic momentum $k=q+f \tilde{u}$,

$$
\begin{aligned}
n_{k}(\tilde{u}) & =n_{T} \int_{0}^{\tilde{u}} \exp \left\{-\left(k^{2}+\epsilon\right) u+k f u^{2}-\frac{1}{3} f^{2} u^{3}\right\} d u \\
& +n_{0}(k-f \tilde{u}) \exp \left\{-\left(k^{2}+\epsilon\right) \tilde{u}+k f \tilde{u}^{2}-\frac{1}{3} f^{2} \tilde{u}^{3}\right\} .
\end{aligned}
$$

Note that the argument of $n_{0}$ is a conserved quantity $k-f \tilde{u}$, cf. also Eq. (9.16).

The transition to the limit $t=\tau_{0} \tilde{u} \rightarrow \infty$ is very simple. After several relaxation times we have the stationary distribution

$$
n(k) \equiv n_{k}(\tilde{u} \rightarrow \infty)=n_{T} \int_{0}^{\infty} \exp \left\{-\left(k^{2}+\epsilon\right) u+k f u^{2}-\frac{1}{3} f^{2} u^{3}\right\} d u
$$

Restoring $k \rightarrow k_{x}, \epsilon \rightarrow \epsilon+w$, and $n(k) \rightarrow n\left(k_{x} ; w, f, \epsilon\right)$ we return to the solution of the static Boltzmann equation Eq. (3.4). Consequently the dummy parameter $t=\tau_{0} u$ in Eq. (2.11) has the meaning of the time interval between the birth of fluctuation Cooper pairs and the moment when we measure the current. Hence the integrand in the formula for the current Eq. (2.15) has the meaning of the part of the current given by Cooper pairs born a time span $t$ before the moment of the measurement. We consider it interesting that according to Eqs. (5.18, 5.19) this "age distribution" has a $\operatorname{sharp} \delta$-like maximum at $t_{0}=\tau_{0} u_{0}$ for the supercooled normal phase in small electric fields. 
Finally, it is amusing to note that although the fluctuation Cooper pairs are neither particles nor quasi-particles the conservation of canonical momentum implies Newton's equation of motion

$$
\frac{d \mathbf{p}}{d t}=e^{*} \mathbf{E}
$$

${ }^{1}$ A. Larkin and A. Varlamov, Fluctuation Phenomena in Superconductors, Sec.4.3 in "Physics of Conventional and Unconventional Superconductors", Edited by K. Bennemann and J. B Ketterson, (Springer, Berlin, to appear in 2002).

${ }^{2}$ W. J. Skocpol and M. Tinkham, Rep. Prog. Phys. 38, 1094 (1975), Sec. 5.3, Fig. 15, and references therein.

3 T. Mishonov and E. Penev, International Journal of Modern Physics B14, 3831-3878 (2000).

${ }^{4}$ L. D. Landau and E. M. Lifshitz, Physical Kinetics, (Pergamon, New York, 1973) Sec.25., Eq. (25.12); A. A. Abrikosov, Fundamentals of the Theory of Metals, (North Holland, Amsterdam, 1988), J. M. Ziman, Principles of the Theory of Solids, (Cambridge Univ Press, 1972) Chap.7; J. M. Ziman, Electrons and Phonons, (Oxford Univ Press, 1960), I. M. Lifshitz, M. I. Kaganov, Sov. Phys. Usp. 2, 831 (1960), ibid 5, 878 (1963), ibid 8, 805 (1966) (Usp. Fiz. Nauk 69, 419 (1959), ibid 78, 411 (1962), ibid 87, 389 (1965)).

${ }^{5}$ N. W. Ashcroft, N. D. Mermin, Solid State Physics, (Holt, Rinehart and Winston, New York, 1976); Chap.16.

${ }^{6}$ T. M. Mishonov, Phys. Rev. Lett. 67, 3195 (1991); A. T. Fiory, A. F. Hebard, R. H. Eick, P. M. Mankiewich, R. E. Howard, M. L. O'Malley, ibid. 67, 3196 (1991).

7 T. Mishonov and N. Zahariev, Superlattices and Microstructures 26, 57-60 (1999).

${ }^{8}$ T. M. Mishonov, Phys. Rev. B 42, 6715 (1990); K. Karrai, E. Choi, F. Dunmore, S. Liu, X. Ying, Qi Li, T. Venkatesan, H. D. Drew, Qi Li, D. B. Fenner, Phys. Rev. Lett. 69, 355 (1992).

9 T. M. Mishonov, Phys. Rev. B 50, 4004 (1994).

10 T. M. Mishonov, Phys. Rev. B 50, 4009 (1994).

${ }^{11}$ T. M. Mishonov and D. Ch. Damianov, Czech. J. Phys. 46, (Suppl. S2) 631 (1996).

12 D. Ch. Damianov and T. M. Mishonov, Superlattices and Microstructures 21, 467 (1997).

${ }^{13}$ L. P. Gor'kov, Zh. Eksp. Teor. Fiz. Pis. Red. 11, 52-56 (1970); (Engl. transl. Sov. Phys.-JETP Lett. 11, 32-35 (1970)); Eq. (5).

14 A. A. Varlamov and L. Reggiani, Phys. Rev. B 45, 1060-1063 (1992); Eqs. (1,6).

15 J. P. Hurault, Phys. Rev. 179, 494-496 (1969).

${ }^{16}$ A. Dorsey, Phys. Rev. B 43, 7575-7585 (1991).

17 A. A. Varlamov, L. Yu, Phys. Rev. B 44, 7078-7080 (1991).

18 A. T. Fiory, A. F. Hebard and W. I. Glaberson, Phys. Rev. B 28, 5075-5087 (1983), Fig. 3. 